\title{
LINEAR SYZYGIES, HYPERBOLIC COXETER GROUPS AND REGULARITY
}

\author{
ALEXANDRU CONSTANTINESCU, THOMAS KAHLE, AND MATTEO VARBARO
}

\begin{abstract}
We show that the virtual cohomological dimension of a Coxeter group is essentially the regularity of the Stanley-Reisner ring of its nerve. Using this connection between geometric group theory and commutative algebra, as well as techniques from the theory of hyperbolic Coxeter groups, we study the behavior of the Castelnuovo-Mumford regularity of square-free quadratic monomial ideals. We construct examples of such ideals which exhibit arbitrarily high regularity after linear syzygies for arbitrarily many steps. We give a doubly logarithmic bound on the regularity as a function of the number of variables if these ideals are Cohen-Macaulay.
\end{abstract}

\section{INTRODUCTION}

The Castelnuovo-Mumford regularity captures the complexity of finitely generated graded $R$-modules, where $R=\mathbb{k}\left[x_{1}, \ldots, x_{n}\right]$ is a standard graded polynomial ring in $n$ variables over a field $\mathbb{k}$. We focus on the case of modules of the kind $R / I$, where $I$ is a homogeneous ideal of $R$. A fundamental question is how big the regularity of $R / I$ can be, when $I \subseteq R$ is generated in fixed degree. The following are some important results in this area.

i) For any $d \geq 2$, Mayr and Meyer [MM82] provided ideals $I \subseteq R$ generated in degrees $\leq d$ for which reg $R / I$ is doubly exponential in the number of variables $n$, as explained by Bayer and Stillman in [BS88].

ii) Caviglia and Sbarra showed in [CS05] that reg $R / I \leq(2 d)^{2^{n-2}}$ provided $I$ is generated in degrees $\leq d$.

iii) Ananyan and Hochster [AH16] proved that, if $I$ is generated by $r$ forms of degrees $\leq d$, then projdim $R / I \leq \phi(r, d)$ provided the characteristic of $\mathbb{k}$ is zero or larger than $d$ (here $\phi$ is a function not depending on the number of variables $n$ ). This solves Stillman's conjecture [PS09] in characteristic zero or bigger than $d$. By a result of Caviglia (see for example [MS13, Theorem 2.4]), projective dimension can be equivalently replaced by regularity in the above statement.

iv) McCullough and Peeva provided in [MP18] examples of homogeneous prime ideals $\mathfrak{p} \subseteq R$ such that $\operatorname{reg} R / \mathfrak{p}$ is not bounded by any polynomial function in the multiplicity. In particular, this shows that the Eisenbud-Goto conjecture [EG84] is false.

2010 Mathematics Subject Classification. Primary: 13F55; 20F55; Secondary: 13D02.

Key words and phrases. Stanley-Reisner ring, simplicial complex, syzygy, hyperbolic Coxeter group, flag-nosquare complex.

Large parts of this research were carried out at Mathematisches Forschungsinstitut Oberwolfach within the Research in Pairs program. The work was continued with support from the MIUR-DAAD Joint Mobility Program (Proj. n. 57267452). 
v) Caviglia, Chardin, McCullough, Peeva and the third named author noticed in $\left[\mathrm{CCM}^{+} 19\right]$ that, if $\mathbb{k}$ is algebraically closed, there exists a function $\phi(e)$ bounding reg $R / \mathfrak{p}$ from above whenever $\mathfrak{p}$ is a homogeneous prime ideal of multiplicity $e$.

The Castelnuovo-Mumford regularity of $R / I$ can be read off the graded Betti numbers $\beta_{i j}$ of $R / I$ as $\operatorname{reg} R / I=\max \left\{j-i: \beta_{i j} \neq 0\right\}$ (see Section 2.4 for preliminaries on commutative algebra). The Mayr-Meyer ideals have the property that $\beta_{2 j} \neq 0$ for a certain $j>d^{2^{n / 10}}$. That is, their eventually high regularity is visible early in the resolution, indicating a possible connection between different homological degrees. Part of the purpose of the present paper is to investigate the possibilities for such connections. Specifically, we study the behavior of the regularity of free resolutions that stay linear until a certain homological degree. As an example for questions concerning the limit behavior of regularity consider the following open problem.

Question 1.1. Is there a family of quadratically generated ideals $\left\{I_{n} \subseteq R=\mathbb{k}\left[x_{1}, \ldots, x_{n}\right]\right\}_{n \in \mathbb{N}}$ with linear syzygies such that

$$
\lim _{n \rightarrow \infty} \frac{\operatorname{reg} R / I_{n}}{n}>0 \quad \text { ? }
$$

Following Green and Lazarsfeld [GL86, Section 3a], we say that, given an integer $p \geq 1, R / I$ satisfies property $N_{p}$ if $\beta_{i j}=0$ for all $1 \leq i \leq p$ and $j \neq i+1$. So $R / I$ satisfies property $N_{1}$ if and only if $I$ is quadratically generated, it satisfies property $N_{2}$ if and only if $I$ is quadratically generated and has linear first syzygies, and so on. The Green-Lazarsfeld index of $R / I$, denoted by index $R / I$, is the largest $p$ such that $R / I$ satisfies $N_{p}$ where by convention, index $R / I=\infty$ if $I$ has a 2-linear resolution, and index $R / I=0$ if $I$ is not quadratically generated. If $I$ is a Mayr-Meyer ideal, then its Green-Lazarsfeld index is at most one.

As a consequence of Eisenbud-Schreyer's construction of pure modules, a syzygy degree that appears in a free resolution can be unrelated to all earlier parts of the resolution [ES09]. A construction due to Ullery shows that for any $p, k \in \mathbb{N}, k>p+1$, there even is a homogeneous ideal $I \subseteq R$ such that $R / I$ satisfies $N_{p}$ and $\beta_{p+1, k} \neq 0$, see [Ull14]. These constructions need a large number of variables in $R$, though, and are not efficient enough for Question 1.1. Due to the flexibility of resolutions of general ideals, it is interesting to look at more restricted classes. For example, Koszul algebras cannot exhibit extremal behaviour as above. It is known, however, that for all $p \geq 2$ there exist families of homogeneous ideals $I_{n} \subset R$ such that $R / I_{n}$ is a Koszul algebra satisfying $N_{p}$ and $\lim _{n \rightarrow \infty}\left(\operatorname{reg} R / I_{n}\right) / \sqrt[p]{n}>0$ [ACI13, Section 6].

In this paper we are interested in monomial ideals. Here the situation is even more rigid as the following result by Dao, Huneke and Schweig [DHS13] illustrates. If $I \subset R$ is a square-free monomial ideal such that $R / I$ satisfies $N_{p}$ for some $p \geq 2$, then

$$
\operatorname{reg} R / I \leq \log _{\frac{p+3}{2}}\left(\frac{n-1}{p}\right)+2 .
$$

In particular, a family which gives a positive answer to Question 1.1 cannot consist of monomial ideals. In Section 4 we derive a new doubly logarithmic bound when $R / I$ is Cohen-Macaulay.

The main motivation for the present paper is the following question:

Question 1.2. Fix an integer $p \geq 2$. Is there a bound $r(p)$ (independent of $n$ ) such that $\operatorname{reg} R / I \leq r(p)$ for all monomial ideals $I \subset R$ such that $R / I$ satisfy $N_{p}$ ? 
For $p=2$, a negative answer has been given by the authors in [CKV16]. If $R / I$ is Gorenstein, the answer is positive by [CKV16, Theorem 4]. If $R / I$ is Cohen-Macaulay, then the answer is unknown (Question 4.4). In this paper we give a negative answer for arbitrary $p$ and begin the search for constructions that realize the negative answer with as few variables as possible.

These investigations lead us to the consideration of a connection between square-free monomial ideals and Coxeter groups. It starts from the observation that square-free monomial ideals with property $N_{2}$ correspond to right-angled hyperbolic Coxeter groups (see Section 2.3.1). The study of the geometry and topology of such groups contains many ideas that we feel can be useful for commutative algebra. In Section 5 we start to develop this connection, proving as a cornerstone the following identity of homological invariants (Theorem 5.2).

$$
\operatorname{vcd} W=\max _{\text {char } \mathbb{k}}\{\operatorname{reg} \mathbb{k}[\mathcal{N}]\},
$$

where $W$ is a Coxeter group with nerve $\mathcal{N}(W)$, vcd $W$ is the virtual cohomological dimension of $W$, and $\mathbb{k}[\mathcal{N}(W)]$ is the Stanley-Reisner ring of the simplicial complex $\mathcal{N}(W)$. As the regularity of a Stanley-Reisner ring depends only on the characteristic of the field, the maximum is taken over all possible characteristics, choosing one field for each.

We see (1) as a general tool to transfer results from Coxeter group theory to combinatorial commutative algebra and vice versa. For example, when $p=2$, Question 1.2 is equivalent to the following question of Gromov:

Question 1.3. Is there a global bound on the virtual cohomological dimension of hyperbolic right-angled Coxeter groups?

In fact, a right-angled Coxeter group $W$ is hyperbolic if and only if $\mathbb{k}[\mathcal{N}(W)]$ satisfies $N_{2}$. As an immediate consequence of (1) and the bound of [DHS13] we get:

Corollary 1.4. If $W$ is a hyperbolic right-angled Coxeter group with $n$ generators, then

$$
\operatorname{vcd} W \leq \log _{\frac{5}{2}}\left(\frac{n-1}{2}\right)+2
$$

Gromov's question had already been answered negatively in [JŚ03]. Later on, new examples were constructed by Osajda in [Osa13b]. Let $2^{(\Delta)}$ denote the face complex of a simplicial complex $\Delta$ (Definition 2.8). Exploiting ideas from Osajda's construction and (1), we prove

Theorem 6.11. Let $I=I_{\Delta} \subseteq R$ be a square-free quadratic monomial ideal. If char $(\mathbb{k})=0$, then there is a positive integer $N$ and a square-free monomial ideal $I^{\prime}=I_{\Delta^{\prime}} \subseteq R^{\prime}=\mathbb{k}\left[y_{1}, \ldots, y_{N}\right]$ such that:

i) $\operatorname{reg} R^{\prime} / I^{\prime}=\operatorname{reg} R / I+1$;

ii) index $R^{\prime} / I^{\prime}=$ index $R / I$;

iii) For each vertex $v$ of $\Delta^{\prime}, \mathrm{lk}_{\Delta^{\prime}} v=2^{(\Delta)}$.

As a corollary, we get a negative answer to Question 1.2:

Corollary 6.12. For any positive integers $p$ and $r$, there exists a square-free monomial ideal $I \subseteq R=\mathbb{k}\left[x_{1}, \ldots, x_{N(p, r)}\right]$, such that $R / I$ satisfies $N_{p}$ and $\operatorname{reg} R / I=r$. 
The proofs of these statements are contained in Section 6. The crux of the corollary is that the number of indeterminates $N(p, r)$ depends on the desired $r$ and $p$. In Section 7 we give an explicit upper bound for the minimal number of variables in the corollary (Theorem 7.4).

The following Section 2 contains some preliminaries that we hope will be useful to readers not already initiated in commutative algebra and geometric group theory. Section 3 gathers some new homological properties of Stanley-Reisner rings inspired by the developments in this paper, but potentially useful beyond. In Section 4 we prove a new doubly logarithmic upper bound on the regularity of Stanley-Reisner rings of complexes with top homology and property $N_{p}$ (Theorem 4.2), which yields the same bound for all Cohen-Macaulay Stanley-Reisner rings with property $N_{p}$ (Corollary 4.3). Section 5 establishes the fundamental equality (1). Finally, Sections 6 and 7 give Theorem 6.11 and an upper bound on the number of variables necessary for arbitrary regularity with property $N_{p}$ (Theorem 7.4).

\section{PRELIMINARIES}

As this paper touches upon the somewhat separated topics of geometric group theory, commutative algebra, and combinatorics, we introduce some preliminaries first.

2.1. Cell complexes. A poset is a partially ordered set $(\mathcal{P}, \leq)$. For every element $p \in \mathcal{P}$ we define the subposets $\mathcal{P}_{\leq p}=\{q \in \mathcal{P}: q \leq p\}$ and $\mathcal{P}_{\geq p}=\{q \in \mathcal{P}: q \geq p\}$. We do not assume that $\mathcal{P}$ is finite.

Definition 2.1. An (abstract) convex cell complex is a poset $\mathcal{P}$ that satisfies the following two conditions:

i) For each $p \in \mathcal{P}$, the subposet $\mathcal{P}_{\leq p}$ is isomorphic to the poset of faces of some finite convex polytope (including the empty face).

ii) For any $p_{1}, p_{2} \in \mathcal{P}$ the poset $\mathcal{P}_{\leq p_{1}} \cap \mathcal{P}_{\leq p_{2}}$ contains a greatest element.

The elements of $\mathcal{P}$ are called faces, and the maximal elements are called facets. If each of the convex polytopes in condition (i) are simplices (respectively cubes), then $\mathcal{P}$ is an abstract simplicial complex (respectively an abstract cubical complex).

Conditions (i) and (ii) imply that, if $\mathcal{P} \neq \emptyset$, it has a unique minimal element $\hat{0}$, and a well defined rank function. The minimal element corresponds to the empty face, and the rank function defines the dimension of a face: $\operatorname{dim}(p)=\operatorname{rank}(p)-1$. The 0 -dimensional faces are called vertices and the 1-dimensional faces are called edges. The 1-skeleton of a complex $\mathcal{P}$ is the subposet of elements of rank at most 2. We also interpret faces as finite sets of vertices: $F=\left\{\right.$ rank 1 elements of $\left.\mathcal{P}_{\leq F}\right\}$. In this interpretation, the partial order is inclusion of sets. This way, a cell complex is a collection of finite subsets of a (possibly infinite) vertex set. A cell complex is thus a simplicial complex if the collection is closed under taking subsets. We can always speak of the cardinality of a face; however, the rank corresponds to the cardinality of faces only for simplicial complexes. Nonfaces are collections of vertices which do not correspond to any face. These can also be ordered by inclusion, and minimal nonfaces are well defined.

A convex cell complex is a cell if it has a unique maximal element $F_{0}$; the boundary of the cell is the poset $\mathcal{P} \backslash\left\{F_{0}\right\}$. The subcomplex of $\mathcal{P}$ induced by a nonempty subset $V$ of its vertex set is $\left.\mathcal{P}\right|_{V}=\bigcup_{p \in V} \mathcal{P}_{\geq p} \cup\{\hat{0}\}$. Some authors use the term full subcomplex for our induced subcomplexes. Not all subcomplexes are induced (e.g. the boundary of the triangle is not an 
induced subcomplex of the triangle, but all edges are induced subcomplexes). A cell complex is locally finite if $\mathcal{P}_{\geq p}$ is a finite poset for every $\hat{0} \neq p \in \mathcal{P}$.

Definition 2.2. Let $\mathcal{P}$ be an abstract convex cell complex and $F \in \mathcal{P}$ a face. The link $\operatorname{lk}_{\mathcal{P}} F$ of $F$ in $\mathcal{P}$ is the abstract convex cell complex $\mathcal{P}_{\geq F}$.

Remark 2.3. If $F$ is a vertex, Definition 2.2 yields what is commonly known as the spherical link at the vertex. Here we prefer a combinatorial definition as we do not think of our complexes as embedded in a metric space.

Remark 2.4. If $\mathcal{P}$ is a cubical or a simplicial complex, then every link is a simplicial complex. If $\mathcal{P}$ is locally finite, then the link is a finite complex.

Example 2.5. The link of each vertex in the 3-dimensional cube is a triangle. The link of each vertex of the octahedron is a square.

2.1.1. Simplicial complexes. It is easy to check that, for simplicial complexes, all the "usual" definitions agree with the ones given above. A simplicial complex $\Delta$ is flag, if all the minimal nonfaces have cardinality two. Equivalently, no induced subcomplex is the boundary of a simplex. For any integer $k \geq 3$, the $k$-cycle is the 1-dimensional simplicial complex with vertex set $\left\{v_{i}\right\}_{i=0, \ldots, k-1}$ and edge set $\left\{\left\{v_{i}, v_{i+1}(\bmod k)\right\}\right\}_{i=0, \ldots, k-1}$. The following property of simplicial complexes is essential to this paper, as it has interpretations in both commutative algebra and Coxeter group theory.

Definition 2.6. Let $k \geq 4$ be an integer. A simplicial complex is $k$-large, if it is flag and does not have any induced $j$-cycles for $j<k$.

A cubical or simplicial complex is locally $k$-large if all its vertex links are $k$-large. In the literature, 5-largeness is sometimes referred to as flag-no-square or Siebenmann's condition. We stress here that all $k$-large complexes must be flag, and that an induced cycle contains no diagonals.

Example 2.7. Let $\Delta$ be the boundary of the octahedron, i.e. $\Delta$ has vertex set $\left\{ \pm v_{i}\right\}_{i=1,2,3}$ and eight 2-dimensional facets: $\left\{ \pm v_{1}, \pm v_{2}, \pm v_{3}\right\}$. This complex is flag, because the minimal nonfaces are $\left\{+v_{i},-v_{i}\right\}$, but it is not 5 -large, because the vertex subset $\left\{ \pm v_{1}, \pm v_{2}\right\}$ induces a 4-cycle. Adding the edges $\left\{+v_{i},-v_{i}\right\}$ for $i=1,2,3$ to $\Delta$, we obtain a simplicial complex without induced 4-cycles, but it is not flag.

Definition 2.8. Let $\Delta$ be a simplicial complex. The face complex $2^{(\Delta)}$ is the simplicial complex whose vertex set is the set of nonempty faces of $\Delta$ and where $F_{1}, \ldots, F_{s} \in \Delta$ form a face of $2^{(\Delta)}$ if and only they are all contained in a single face of $\Delta$.

Example 2.9. The face complex of a $d$-simplex is the $\left(2^{d+1}-2\right)$-simplex.

2.2. Coxeter groups. We use the notation from Davis' book [Dav08]. A Coxeter system is a pair $(W, S)$ consisting of a finitely generated group $W$ and a finite set of distinct generators $S=\left\{s_{1}, \ldots, s_{n}\right\}$, all different from the identity, such that $W$ is presented as

$$
W=\left\langle s_{1}, \ldots, s_{n}:\left(s_{i} s_{j}\right)^{m_{i j}}=e\right\rangle
$$

for $m_{i j} \in \mathbb{N} \cup\{\infty\}$ with $m_{i i}=1$, and $m_{i j} \geq 2$ for $i \neq j$. The case $m_{i j}=\infty$ means no relation. If a group has a presentation as above, then it is a Coxeter group, and $S$ is a set of Coxeter 
generators. The finite Coxeter groups have been classified by Coxeter [Cox35]. The matrix $M=\left(m_{i j}\right)_{i j}$ is the Coxeter matrix of $(W, S)$. If $m_{i j} \in\{1,2, \infty\}$, then the Coxeter group (or Coxeter system) is right-angled. The elements of $S$ are letters, and the elements of $W$ are words.

A special subgroup of $W$ is a subgroup $W_{T}$ generated by a subset $T \subseteq S$ of the Coxeter generators. In particular, the trivial subgroup is special. By [Dav08, Theorem 4.1.6], $\left(W_{T}, T\right)$ is a Coxeter system for all $T \subset S$. A subset $T \subseteq S$ is spherical if $W_{T}$ is finite. In this case, $W_{T}$ and the words in it are also called spherical. A spherical coset is a coset of a spherical subgroup. All spherical cosets are finite. Clearly, being spherical is closed under taking subsets.

Definition 2.10. The nerve $\mathcal{N}(W, S)$ of a Coxeter system $(W, S)$ is the simplicial complex consisting of the spherical sets ordered by inclusion.

The nerve of a Coxeter system is always a finite simplicial complex, with the Coxeter generators as vertices.

Remark 2.11. There is a one-to-one correspondence between right-angled Coxeter groups and flag simplicial complexes given as follows. Every flag simplicial complex $\Delta$ is the nerve of a right-angled Coxeter group $\mathcal{W}(\Delta)$ : the off-diagonal entries of the Coxeter matrix of $\mathcal{W}(\Delta)$ are $m_{i j}=2$ whenever $\{i, j\} \in \Delta$ and $m_{i j}=\infty$ otherwise. Conversely, if $(W, S)$ is right-angled, and $T \subseteq S$, such that any two elements are connected by an edge in the nerve, then $W_{T} \cong(\mathbb{Z} / 2 \mathbb{Z})^{|T|}$.

Remark 2.12. In a right-angled Coxeter group a word is spherical if and only if it can be written with letters that commute pairwise. In particular, if the presentation is reduced (i.e. no subword is equal to the word), then each letter appears at most once.

Example 2.13. Not every simplicial complex is the nerve of a Coxeter system. The smallest counterexample occurs on five vertices and is given by the complex with facets $\{123,145,245$, $345\}$. This can be confirmed using the classification of finite Coxeter groups.

Definition 2.14. The Davis complex of a Coxeter system $(W, S)$ is the cell complex $\Sigma(W, S)$ given by the poset of spherical cosets.

Remark 2.15. The link of any vertex $w$ of $\Sigma(W, S)$ is the poset of spherical cosets $w W_{T}$ for all spherical subsets $T$. It is thus isomorphic to the nerve $\mathcal{N}(W, S)$.

Remark 2.16. Davis and Januszkiewicz have discovered a link between Stanley-Reisner theory and Coxeter groups that is different from the developments in our paper. The cohomology ring $H^{*}\left(W, \mathbb{F}_{2}\right)$ is isomorphic to the Stanley-Reisner ring $\mathbb{F}_{2}[\mathbb{N}(W, S)]$. However, this connection is a characteristic two phenomenon, as otherwise the product in the cohomology ring need not be commutative. See [DJ91, Theorem 4.11].

2.3. Geometric group theory. Let $\Gamma$ be a simple graph on a (possibly infinite) vertex set $V$. Given two vertices $v, w \in V$, a path $e$ from $v$ to $w$ is a subset $\left\{v=v_{0}, v_{1}, v_{2}, \ldots, v_{k}=w\right\} \subseteq V$, such that $\left\{v_{i}, v_{i+1}\right\}$ is an edge for all $i=0, \ldots, k-1$. The length of a path is $\ell(e)=k$. The distance between $v$ and $w$ is

$$
d(v, w):=\min \{\ell(e): e \text { is a path from } v \text { to } w\} .
$$

If $W \subset V$ is a set of vertices, then $d(v, W):=\min \{d(v, w), w \in W\}$. A path $e$ from $v$ to $w$ is a geodesic path if $\ell(e)=d(v, w)$. A geodesic triangle of vertices $v_{1}, v_{2}$, and $v_{3}$ consists of three 
geodesic paths $e_{i}$ from $v_{i}$ to $v_{i+1(\bmod 3)}$ for $i=1,2,3$. For a real number $\delta \geq 0$, a geodesic triangle $e_{1}, e_{2}, e_{3}$ is $\delta$-slim if $d\left(v, e_{i} \cup e_{j}\right) \leq \delta$ for all $v \in e_{k}$ and $\{i, j, k\}=\{1,2,3\}$. The graph $\Gamma$ is $\delta$-hyperbolic if each geodesic triangle of $\Gamma$ is $\delta$-slim, and hyperbolic if it is $\delta$-hyperbolic for some $\delta \geq 0$.

2.3.1. Hyperbolic groups. Let $G$ be a group and $S$ a set of distinct generators of $G$, not containing the identity. The Cayley graph $\operatorname{Cay}(G, S)$ is the simple graph with vertex set $G$ and edges $\{g, g s\}$ for all $g \in G$ and $s \in S$. For example, the vertices of the Davis complex $\Sigma(W, S)$ of a Coxeter system $(W, S)$ are the elements of $W$ and the edges are the cosets of the spherical subgroups $W_{s_{i}}$. Therefore the 1 -skeleton of $\Sigma(W, S)$ is the Cayley graph of $(W, S)$. Gromov proved that if Cay $(G, S)$ is hyperbolic for some finite set of generators $S$ then it is hyperbolic for any finite set of generators $S$ [Dav08, Theorem 12.3.5].

This justifies the definition of hyperbolic groups in the following way.

Definition 2.17. A group $G$ is hyperbolic if $\operatorname{Cay}(G, S)$ is a hyperbolic graph for some (equivalently for any) finite set of generators $S$.

It is easy to check that $\mathbb{Z}^{2}$ is not hyperbolic. Therefore, if $G$ contains $\mathbb{Z}^{2}$ as a subgroup, then $G$ cannot be hyperbolic. By work of Moussong, for a Coxeter group $(W, S)$ this can be reversed:

$$
W \text { is hyperbolic } \Longleftrightarrow \mathbb{Z}^{2} \not \subset W \text {. }
$$

Combining results of Siebenmann [Dav08, Lemma I.6.5] and Moussong [Dav08, Lemma 12.6.2], if $(W, S)$ is right-angled then

$$
W \text { is hyperbolic } \Longleftrightarrow \mathcal{N}(W, S) \text { has no induced 4-cycles. }
$$

\subsubsection{Cohomological dimension. The cohomological dimension of a group $G$ is}

$$
\operatorname{cd} G=\sup \left\{n: H^{n}(G ; M) \neq 0 \text { for some } \mathbb{Z} G \text {-module } M\right\},
$$

where $H^{n}(G ; M)$ is the $n$-th group cohomology of $G$ with values in $M$ (see [Dav08, Appendix F] for equivalent definitions and some properties). If $G$ has nontrivial torsion, then $\operatorname{cd} G=+\infty$ (see [Dav08, Lemma F.3.1]). Therefore the notion is not interesting for groups with torsion, but this can be rectified. A group $G$ is virtually torsion-free if it has a finite index subgroup which is torsion-free. It follows from a result of Serre [Dav08, Theorem F.3.4] that, if $\Gamma$ and $\Gamma^{\prime}$ are two finite index torsion-free subgroups of $G$, then $\operatorname{cd} \Gamma=\operatorname{cd} \Gamma^{\prime}$. Thus the following notion is well-defined.

Definition 2.18. Let $G$ be a virtually torsion-free group, and $\Gamma$ some (equivalently any) finite index torsion-free subgroup of $G$. The virtual cohomological dimension of $G$ is

$$
\operatorname{vcd} G=\operatorname{cd} \Gamma .
$$

Each nontrivial Coxeter group has torsion but, admitting a faithful linear representation (see [Dav08, Corollary D.1.2]), it is virtually torsion-free. Thus the virtual cohomological dimension is always well-defined for a Coxeter group. By [Dav08, Corollary 8.5.5], and using [Mun84, Lemma 70.1] to avoid geometric realizations, the vcd of a Coxeter group $(W, S)$ can be read off the nerve $\mathcal{N}(W, S)$, namely:

$$
\operatorname{vcd} W=\max \left\{i: \widetilde{H}^{i-1}(\mathcal{N}(W, S) \backslash \sigma ; \mathbb{Z}) \neq 0 \text { for some } \sigma \in \mathcal{N}(W, S)\right\},
$$


where $\mathcal{N}(W, S) \backslash \sigma$ is the restriction of $\mathcal{N}(W, S)$ to $S \backslash \sigma$, and $\widetilde{H}^{i}$ denotes the reduced simplicial cohomology modules.

2.4. Commutative Algebra. Let $n$ be a positive integer, $R=\mathbb{k}\left[x_{1}, \ldots, x_{n}\right]$ the polynomial ring in $n$ variables over a field $\mathbb{k}$, and $\mathfrak{m}=\left(x_{1}, \ldots, x_{n}\right)$ its irrelevant ideal. Any quotient $R / I$ by some homogeneous ideal $I \subseteq R$ has a minimal graded free resolution.

$$
0 \rightarrow \bigoplus_{j \in \mathbb{Z}} R(-j)^{\beta_{k j}} \rightarrow \cdots \rightarrow \bigoplus_{j \in \mathbb{Z}} R(-j)^{\beta_{2 j}} \rightarrow \bigoplus_{j \in \mathbb{Z}} R(-j)^{\beta_{1 j}} \rightarrow R \rightarrow R / I \rightarrow 0 .
$$

The Betti number $\beta_{i j}$ is the number of minimal generators of degree $j$ of the free module in homological degree $i$ in the resolution. It is independent of the particular minimal resolution and can be computed as $\beta_{i, j}(R / I)=\operatorname{dim}_{\mathbb{k}} \operatorname{Tor}_{i}(R / I, \mathbb{k})_{j}$.

Definition 2.19. The Castelnuovo-Mumford regularity of $R / I$ is

$$
\operatorname{reg}(R / I)=\max \left\{j-i: \beta_{i, j}(R / I) \neq 0\right\}
$$

If $H_{\mathfrak{m}}^{i}$ denotes local cohomology with support in $\mathfrak{m}$, [Eis95, Proposition 20.16] and Grothendieck duality imply $\operatorname{reg}(R / I)=\max \left\{j+i: H_{\mathfrak{m}}^{i}(R / I)_{j} \neq 0\right\}$,

Definition 2.20. For any positive integer $p$, the $\mathbb{k}$-algebra $R / I$ satisfies property $N_{p}$ if:

$$
\beta_{i, j}(R / I)=0 \quad \forall i=1, \ldots, p \text { and } j \neq i+1 .
$$

Definition 2.21. Let $\Delta$ be a finite simplicial complex with vertex set $[n]=\{1, \ldots, n\}$. The Stanley-Reisner ring of $\Delta$, denoted by $\mathbb{k}[\Delta]$, is the quotient of $R$ by the square-free monomial ideal

$$
I_{\Delta}=\left(\prod_{i \in A} x_{i}: A \subseteq[n] \text { and } A \notin \Delta\right) .
$$

The ideal $I_{\Delta}$ is the Stanley-Reisner ideal of $\Delta$.

There is a one-to-one correspondence between simplicial complexes and ideals generated by square-free monomials. From the definition it follows that a simplicial complex is flag if and only if its Stanley-Reisner ideal is quadratic. The $N_{p}$ property for Stanley-Reisner rings was characterized combinatorially in [EGHP05, Theorem 2.1]:

Theorem 2.22. The Stanley-Reisner ring $\mathbb{k}[\Delta]$ satisfies $N_{p}$ if and only if $\Delta$ is $(p+3)$-large.

The Castelnuovo-Mumford regularity of $\mathbb{k}[\Delta]$ can be computed from the reduced singular cohomology of either induced subcomplexes or links of $\Delta$. More precisely, Hochster's formula for graded Betti numbers [MS05, Corollary 5.12] gives

$$
\operatorname{reg} \mathbb{k}[\Delta]=\max \left\{i: \widetilde{H}^{i-1}\left(\left.\Delta\right|_{A} ; \mathbb{k}\right) \neq 0 \text { for some } A \subseteq[n]\right\} .
$$

On the other hand, by Hochster's formula for local cohomology [MS05, Theorem 13.13]:

$$
\operatorname{reg} \mathbb{k}[\Delta]=\max \left\{i: \widetilde{H}^{i-1}\left(\mathrm{lk}_{\Delta} \sigma ; \mathbb{k}\right) \neq 0 \text { for some } \sigma \in \Delta\right\} .
$$




\section{Homological Remarks on Stanley-Reisner Rings}

In this section, $\Delta$ is a $d$-dimensional simplicial complex on $n$ vertices, and a face of $\Delta$ is identified with its set of vertices. We use some standard algebraic topology (see for example [Mun84, §5]). For $r \leq d$ let $C_{r}(\Delta ; \mathbb{k})$ be the $\mathbb{k}$-vector space spanned by the $r$-dimensional faces of $\Delta$. Let $\partial_{r}: C_{r}(\Delta ; \mathbb{k}) \rightarrow C_{r-1}(\Delta ; \mathbb{k})$ be the boundary operator and $Z_{r}(\Delta ; \mathbb{k})=\operatorname{Ker} \partial_{r}$ the subspace spanned by the cycles in $C_{r}(\Delta ; \mathbb{k})$. Also write $B_{r}(\Delta ; \mathbb{k})=\operatorname{im} \partial_{r+1}$ for the subspace spanned by the boundaries in $C_{r}(\Delta ; \mathbb{k})$. An $r$-cycle $C$ is nontrivial if $C \notin B_{r}(\Delta ; \mathbb{k})$. A nontrivial $r$-cycle $C$ is vertex-minimal if there is no nontrivial $r$-cycle $C^{\prime}$ with $V\left(C^{\prime}\right) \subsetneq V(C)$, where for $C=\sum_{i=1}^{l} c_{i} F_{i} \in C_{r}(\Delta ; \mathbb{k})$ we set $V(C)=\cup_{i=1}^{l} F_{i}$. For every $v \in V(C)$ define

$$
C_{v}=\sum_{F_{i} \ni v} c_{i}\left(F_{i} \backslash v\right) \in C_{r-1}\left(\mathrm{lk}_{\Delta} v ; \mathbb{k}\right)
$$

If $C \in Z_{r}(\Delta ; \mathbb{k})$, then all codimension one faces containing $v$ sum to zero when applying $\partial$, so $\partial\left(\sum_{F_{i} \ni v} c_{i} F_{i}\right)=C_{v}$; therefore $\partial\left(C_{v}\right)=\partial^{2}\left(\sum_{F_{i} \ni v} c_{i} F_{i}\right)=0$ in $C_{r-2}(\Delta ; \mathbb{k})$ and thus, since the differentials of $\left(C_{i}\left(\mathrm{lk}_{\Delta} v ; \mathbb{k}\right)\right)_{i}$ are just the restrictions of the differentials of $\left(C_{i}(\Delta ; \mathbb{k})\right)_{i}$, we get $C_{v} \in Z_{r-1}\left(\mathrm{lk}_{\Delta} v ; \mathbb{k}\right)$.

Lemma 3.1. Let $C=\sum_{i=1}^{l} c_{i} F_{i}$ be a nontrivial r-cycle in $\Delta$ and $v \in V(C)$ a vertex.

i) If $r=d$, then $C_{v}$ is a nontrivial $(d-1)$-cycle in $\mathrm{lk}_{\Delta} v$.

ii) If $C$ is vertex-minimal, then $C_{v}$ is a nontrivial $(r-1)$-cycle in $\left.\left(\mathrm{lk}_{\Delta} v\right)\right|_{V(C)}$.

Proof. i) is clear. For ii), it is harmless to assume that, in the linear order given to the vertices of $\Delta, v$ comes first in $V(C)$. Assume there exists $B_{v}=b_{1} G_{1}+\ldots+b_{s} G_{s}$ with $b_{i} \in \mathbb{k}$, such that the $G_{i}$ are $r$-dimensional faces in $\left.\left(\mathrm{lk}_{\Delta} v\right)\right|_{V(C)}$ and $\partial\left(B_{v}\right)=C_{v}$. Consider the $\mathbb{k}$-linear combination of $(r+1)$-faces in $\Delta$ defined as $B=b_{1}\left(G_{1} \cup v\right)+\ldots+b_{s}\left(G_{s} \cup v\right)$. Then

$$
\partial(B)=-\sum_{F_{i} \ni v} c_{i} F_{i}+B_{v}
$$

So $A=C+\partial(B)$ is a nontrivial $r$-cycle of $\Delta$ (otherwise $C$ would be trivial). However, $v \notin$ $V(A) \subset V(C)$ - a contradiction.

Proposition 3.2. There exists a vertex $v \in \Delta$ such that

$$
\operatorname{reg} \mathbb{k}\left[\mathrm{lk}_{\Delta} v\right] \geq \operatorname{reg} \mathbb{k}[\Delta]-1 .
$$

Proof. Let $V^{\prime}$ be a subset of the vertex set of $\Delta$ such that $\Gamma=\left.\Delta\right|_{V^{\prime}}$ has nontrivial $r$ th homology with coefficients in $\mathbb{k}$, where $\operatorname{reg} \mathbb{k}[\Delta]=r+1$. Let $C$ be a vertex-minimal nontrivial $r$-cycle of $\Gamma$. By Lemma $3.1 \mathrm{ii}), C_{v}$ is a nontrivial $(r-1)$-cycle in $\left.\left(\mathrm{lk}_{\Gamma} v\right)\right|_{V(C)}$, for all $v \in V(C)$. Since $\left.\left(\mathrm{lk}_{\Gamma} v\right)\right|_{V(C)}=\left.\left(\mathrm{lk}_{\Delta} v\right)\right|_{V(C)}$, the proposition follows.

Proposition 3.3. If $I \subseteq R$ is a homogeneous (not necessarily monomial) ideal such that $\sqrt{I}$ is a square-free monomial ideal, then for any $i \in \mathbb{N}, j \in \mathbb{Z}$ the map of $\mathbb{k}$-vector spaces

$$
H_{\mathfrak{m}}^{i}(R / I)_{j} \rightarrow H_{\mathfrak{m}}^{i}(R / \sqrt{I})_{j}
$$

is surjective. In particular, $\operatorname{reg} R / \sqrt{I} \leq \operatorname{reg} R / I$ and $\operatorname{projdim} R / \sqrt{I} \leq \operatorname{projdim} R / I$. 
Proof. Let $A$ denote $R / I$ localized at $\mathfrak{m}$. If char $\mathbb{k}>0$, then the quotient by the nilradical $A_{\text {red }}=A / \sqrt{(0)}$ is $F$-pure. By [Sch09, Theorem 6.1], in characteristic zero, $A_{\text {red }}$ is DuBois.

So in each case, by [MSS17, Lemma 3.3, Remark 3.4] the map

$$
H_{\mathfrak{m}}^{i}(R / I)=H_{\mathfrak{m} A}^{i}(A) \rightarrow H_{\mathfrak{m} A}^{i}\left(A_{\mathrm{red}}\right)=H_{\mathfrak{m}}^{i}(R / \sqrt{I})
$$

is surjective for any $i \in \mathbb{N}$. Since the above map is homogeneous we conclude.

Given two polynomial rings $R=\mathbb{k}\left[x_{1}, \ldots, x_{n}\right]$ and $R^{\prime}=\mathbb{k}\left[y_{1}, \ldots, y_{m}\right]$, a map of $\mathbb{k}$-algebras $f: R \rightarrow R^{\prime}$ is a monomial map if $f\left(x_{i}\right)$ is a monomial in $\left\{y_{1}, \ldots, y_{m}\right\}$ for all $i=1, \ldots, n$.

Lemma 3.4. If $f: R \rightarrow R^{\prime}$ is a monomial map and $I \subseteq R$ is a monomial ideal, then

$$
\operatorname{projdim} R^{\prime} / \sqrt{f(I) R^{\prime}} \leq \operatorname{projdim} R / I \text {. }
$$

Proof. Since $\sqrt{I}$ is a square-free monomial ideal, $\operatorname{projdim} R / I \geq \operatorname{projdim} R / \sqrt{I}$ by Proposition 3.3. By a classical result of Lyubeznik (see the main theorem of [Lyu84]) the projective dimension of $R / \sqrt{I}$ equals $\operatorname{cd}(R, I)$, the cohomological dimension of $I$. Since the computation of local cohomology is independent of the base ring [BS13, Theorem 4.2.1], $\operatorname{cd}\left(R^{\prime}, f(I) R^{\prime}\right) \leq$ $\operatorname{cd}(R, I)$. Again using [Lyu84], $\operatorname{cd}\left(R^{\prime}, f(I) R^{\prime}\right)=\operatorname{projdim} R^{\prime} / \sqrt{f(I) R^{\prime}}$.

Proposition 3.5. If $2^{(\Delta)}$ is the face complex of $\Delta$, then $\operatorname{reg} \mathbb{k}[\Delta]=\operatorname{reg} \mathbb{k}\left[2^{(\Delta)}\right]$.

Proof. Clearly $\operatorname{reg} \mathbb{k}[\Delta] \leq \operatorname{reg} \mathbb{k}\left[2^{(\Delta)}\right]$ by Definition 2.8 and (4). Let $\Gamma$ and $\Gamma^{\prime}$ be the Alexander duals of, respectively, $\Delta$ and $2^{(\Delta)}$. Then, by the Eagon-Reiner theorem [MS05, Theorem 5.63], $\operatorname{projdim} \mathbb{k}[\Gamma]-1=\operatorname{reg} \mathbb{k}[\Delta]$ and projdim $\mathbb{k}\left[\Gamma^{\prime}\right]-1=\operatorname{reg} \mathbb{k}\left[2^{(\Delta)}\right]$. It can be checked that $I_{\Gamma}$ is the following ideal of $R=\mathbb{k}\left[x_{1}, \ldots, x_{n}\right]$ :

$$
I_{\Gamma}=\left(\prod_{i \in[n] \backslash \tau} x_{i}: \tau \text { is a facet of } \Delta\right) .
$$

For $I_{\Gamma^{\prime}} \subseteq R^{\prime}=\mathbb{k}\left[y_{\sigma}: \sigma \in \Delta\right]$ it holds that

$$
I_{\Gamma^{\prime}}=\left(\prod_{\substack{\sigma \in \Delta \\ \sigma \unrhd \tau}} y_{\sigma}: \tau \text { is a facet of } \Delta\right) .
$$

The map $R \stackrel{f}{\rightarrow} R^{\prime}$ defined by $x_{i} \mapsto \prod_{\sigma \in \Delta, i \in \sigma} y_{\sigma}$ gives $I_{\Gamma^{\prime}}=\sqrt{f\left(I_{\Gamma}\right) R^{\prime}}$, so that the result follows from Lemma 3.4.

\section{Regularity From top homology}

The main result of this section is an improvement of the [DHS13] bound in the case that $\Delta$ is a Cohen-Macaulay complex. In this case, a doubly logarithmic bound for the regularity as a function of the number of vertices is possible (Corollary 4.3). The underlying Theorem 4.2 uses similar techniques as the proof of [CKV16, Theorem 7]. We use the following technical lemma, the proof of which is a routine computation using the inequality $(i-1)(i+1)<i^{2}$ several times. 
Lemma 4.1. For any integer $k \geq 3$ we have

$$
\prod_{i=0}^{k-3}(k-i)^{2^{i}}<12^{2^{k-3}}
$$

Theorem 4.2. Let $\Delta$ be a simplicial complex of dimension $d$ on $n$ vertices that is $(p+3)$-large for some $p \geq 2$, and has nontrivial top homology. If $f_{i}(\Delta)$ is the number of $i$-dimensional faces of $\Delta$, then

$$
f_{d}(\Delta)>\left(\frac{p^{2}+6 p+9}{12}\right)^{2^{d-2}} \quad \text { and } \quad f_{0}(\Delta)>\left(\frac{p^{2}+6 p+9}{12}\right)^{2^{d-3}} .
$$

Proof. For every $d$-dimensional simplicial complex $\Delta$ with nontrivial top homology we define

$$
\begin{aligned}
& v_{d}(\Delta)=\min \{\text { number of vertices in a top-dimensional cycle in } \Delta\}, \\
& s_{d}(\Delta)=\min \{\text { number of facets in a top-dimensional cycle in } \Delta\} .
\end{aligned}
$$

Minimizing over all $d$-dimensional $(p+3)$-large complexes with nontrivial top homology, let

$$
\begin{aligned}
& v_{d}=\min \left\{v_{d}(\Delta): \Delta(p+3) \text {-large, with nontrivial top homology }\right\}, \\
& s_{d}=\min \left\{s_{d}(\Delta): \Delta(p+3) \text {-large, with nontrivial top homology }\right\} .
\end{aligned}
$$

This implies in particular that $v_{1}=s_{1}=p+3$.

Fix a complex $\Delta$ satisfying the hypotheses of the theorem. Let $C \in \Delta$ be a top-dimensional cycle with $s_{d}(\Delta)$ facets. For every vertex $v \in C$, the link $\mathrm{lk}_{\Delta} v$ is a $(d-1)$-dimensional simplicial complex with nontrivial top homology by item i) in Lemma 3.1. Furthermore, $\operatorname{lk}_{\Delta} v$ is $(p+3)$ large. Counting codimension one faces in $\left.\Delta\right|_{V(C)}$ with multiplicity, we get:

$$
s_{d}(\Delta) \geq \frac{1}{d+1} \sum_{v \in V(C)} s_{d-1}\left(\mathrm{lk}_{\left.\Delta\right|_{V(C)}} v\right) .
$$

Fix $v \in V(C)$. Every facet $F$ of the link of $v$ in $\left.\Delta\right|_{V(C)}$ is contained in at least two facets of $C$ only one of which can contain $v$. Thus, a map associating to $F \in \mathrm{lk}_{\Delta_{V_{V(C)}}} v$ a vertex $w \neq v$, with $F \cup\{w\} \in \Delta$ is well defined:

$$
\Phi_{v}: \mathcal{F}\left(\mathrm{lk}_{\left.\Delta\right|_{V(C)}} v\right) \longrightarrow V(\Delta) \backslash V\left(\operatorname{star}_{\left.\Delta\right|_{V(C)}} v\right)
$$

We claim that $\Phi_{v}$ is injective. To see this, let $F_{1}, F_{2} \in \mathcal{F}\left(\mathrm{lk}_{\Delta_{V_{V}(C)}} v\right)$ be distinct faces such that $F_{1} \cup\{w\}$ and $F_{2} \cup\{w\}$ are faces of $\Delta$. Since $\Delta$ is flag, there exist $v_{1} \in F_{1}$ and $v_{2} \in F_{2}$ such that $v, v_{1}, w, v_{2}, v$ is a 4 -cycle and $\left\{v_{1}, v_{2}\right\} \notin \Delta$. Since $\operatorname{lk}_{\left.\Delta\right|_{V(C)}} v$ is flag, also $\{v, w\} \notin \Delta$. Because of this contradiction, $\Phi_{v}$ is injective. The injectivity yields $v_{d}(\Delta) \geq s_{d-1}+v_{d-1}+1$ and then, putting together the above inequalities,

$$
s_{d}>\frac{s_{d-1}^{2}}{d+1}, \quad v_{d}>s_{d-1} .
$$

Now, since $s_{1}=p+3$,

$$
s_{d}>\frac{(p+3)^{2^{d-1}}}{\prod_{i=0}^{d-2}(d+1-i)^{2^{i}}} .
$$


Finally, by Lemma 4.1,

$$
f_{d}(\Delta) \geq s_{d}>\frac{(p+3)^{2^{d-1}}}{12^{2^{d-2}}}=\left(\frac{p^{2}+6 p+9}{12}\right)^{2^{d-2}} .
$$

Corollary 4.3. Let $I \subseteq R$ be a square-free monomial ideal such that $R / I$ is a Cohen-Macaulay ring satisfying property $N_{p}$, for $p \geq 2$. Then

$$
\operatorname{reg} R / I \leq \log _{2} \log _{\frac{p^{2}+6 p+9}{12}} n+3 .
$$

Proof. Let $\Delta$ be a simplicial complex on $n$ vertices such that $I=I_{\Delta}$. By Hochster's formula for local cohomology [MS05, Theorem 13.13],

$$
\operatorname{reg} \mathbb{k}[\Delta]=\max \left\{i: \widetilde{H}^{i-1}\left(\mathrm{lk}_{\Delta} \sigma ; \mathbb{k}\right) \neq 0: \sigma \in \Delta\right\} .
$$

Let $\sigma \in \Delta$ attain the maximum. Because $\Delta$ is Cohen-Macaulay, $\operatorname{lk}_{\Delta} \sigma$ has nontrivial top homology. Therefore reg $\mathbb{k}[\Delta]-1=\operatorname{dim} \mathrm{lk}_{\Delta} \sigma=: d$. Since $\Delta$ is $(p+3)$-large, so is $\operatorname{lk}_{\Delta} \sigma$. Hence by Theorem 4.2

$$
n \geq f_{0}\left(\mathrm{lk}_{\Delta} \sigma\right)>\left(\frac{p^{2}+6 p+9}{12}\right)^{2^{d-3}}
$$

and the conclusion follows.

Corollary 4.3 motivates to ask Question 1.2 again with a Cohen-Macaulay restriction. In this case the answer is not known.

Question 4.4. Fix an integer $p \geq 2$. Is there a global bound $r(p)$ (independent of $n$ ) such that $\operatorname{reg} R / I \leq r(p)$ for all monomial ideals $I \subset R$ for which $R / I$ satisfies $N_{p}$ and is CohenMacaulay?

\section{Virtual COHOMOlogical Dimension MEets REGUlarity}

The main theorem of this section establishes a new connection between Coxeter groups and commutative algebra. Its proof is by a cohomology computation using two spectral sequences associated to a double complex. A reference and our source of notation is [GM03, Chapter III].

Fix a ring $A$. For any finite double complex $L=\left(L^{p, q}\right)_{(p, q) \in \mathbb{N}^{2}}$ of $A$-modules, there are two spectral sequences both converging to the cohomology of the diagonal complex $S L$ of $L$, whose entries are $S L^{n}=\oplus_{p+q=n} L^{p, q}$. We denote these spectral sequences by $\left({ }^{I} E_{r}^{p, q}\right)$ and $\left({ }^{I I} E_{r}^{p, q}\right)$. Both converge to ${ }^{I} E^{k}={ }^{I I} E^{k}=H^{k}(S L)$. By [GM03, III.7, Proposition 10], ${ }^{I} E_{2}^{p, q}$ is isomorphic to $H_{I}^{p}\left(H_{I I}^{\bullet, q}\left(L^{\bullet \bullet \bullet}\right)\right)$ (vertical cohomology of horizontal cohomology), while ${ }^{I I} E_{2}^{p, q}$ is isomorphic to $H_{I I}^{p}\left(H_{I}^{q, \bullet}\left(L^{\bullet, \bullet}\right)\right)$ (horizontal cohomology of vertical cohomology).

For alignment with existing notation it is convenient to let $\Delta$ be a simplicial complex with $n+1$ vertices $V=\{0, \ldots, n\}$. For any $s<n$ and any $i \in\{0, \ldots, s\}$, denote by $\Delta^{i}=\left.\Delta\right|_{V \backslash\{i\}}$. For any sequence of integers $0 \leq a_{0}<\ldots<a_{p} \leq s$, let

$$
\Delta^{a_{0}, \ldots, a_{p}}=\bigcap_{k=0}^{p} \Delta^{a_{k}}
$$

Then $\Delta^{a_{0}, \ldots, a_{p}}$ equals the induced subcomplex $\left.\Delta\right|_{V \backslash\left\{a_{0}, \ldots, a_{p}\right\}}$. The first notation, however, is more natural in following. For example, if $\{0, \ldots, s\}$ is not a face of $\Delta$, then $\left\{\Delta^{i}\right\}_{i=0, \ldots, s}$ 
forms a closed cover of $\Delta$, that is $\cup_{i=0}^{s} \Delta^{i}=\Delta$. Denote by $\mathbf{C}^{\bullet}(\Delta, A)$ the cochain complex of a simplicial complex $\Delta$ with coefficients in the ring $A$. Consider the double complex of $A$-modules $\mathcal{C}(A)=\left(\mathcal{C}^{p, q}(A)\right)_{(p, q) \in \mathbb{N}^{2}}$ with

$$
\mathcal{C}^{p, q}(A)=\bigoplus_{a_{0}<\ldots<a_{p}} \mathbf{C}^{q}\left(\Delta^{a_{0}, \ldots, a_{p}} ; A\right), \quad 0 \leq p \leq s, \quad 0 \leq q \leq \operatorname{dim} \Delta .
$$

where the direct sum runs over all sequences of $p+1$ integers $0 \leq a_{0}<\ldots<a_{p} \leq s$. Throughout we use the standard convention that all modules with indices outside of defined bounds are zero. The vertical maps $\mathcal{C}^{p, q}(A) \rightarrow \mathcal{C}^{p, q+1}(A)$ are just the maps defined for each direct summand in the cochain complex $\mathbf{C}\left(\Delta^{a_{0}, \ldots, a_{p}}, A\right)$. The rows

$$
0 \rightarrow \mathcal{C}^{0, \bullet}(A) \stackrel{d^{1}}{\rightarrow} \mathcal{C}^{1, \bullet}(A) \stackrel{d^{2}}{\longrightarrow} \cdots \stackrel{d^{s-1}}{\longrightarrow} \mathcal{C}^{s-1, \bullet}(A) \stackrel{d^{s}}{\rightarrow} \mathcal{C}^{s, \bullet}(A) \rightarrow 0,
$$

are defined by mapping an element $\alpha=\left(\alpha_{a_{0}, \ldots, a_{p}}\right)_{a_{0}<\ldots<a_{p}} \in \mathcal{C}^{p, q}(A)$, to $d^{p+1}(\alpha) \in \mathcal{C}^{p+1, q}(A)$, whose $\left(b_{0}, \ldots, b_{p+1}\right)$ th component is

$$
\left.\sum_{k=0}^{p+1}(-1)^{k}\left(\alpha_{b_{0}, \ldots, \widehat{b_{k}}, \ldots, b_{p+1}}\right)\right|_{\mathbf{C}^{q}\left(\Delta^{\left.b_{0}, \ldots, b_{p+1}\right)}\right.} .
$$

A routine computation confirms that this defines a double complex. The vertical cohomology is by definition the direct sum of the cohomologies of the corresponding $\Delta^{a_{0}, \ldots, a_{p}}$. The horizontal cohomology is nontrivial only in cohomological degree 0 according to the following lemma, whose proof is standard.

Lemma 5.1. For each $q \in\{0, \ldots, \operatorname{dim} \Delta\}$ we have

$$
H^{p}\left(\mathcal{C}^{\bullet, q}(A)\right)= \begin{cases}\mathbf{C}^{q}\left(\cup_{i} \Delta^{i} ; A\right) & \text { if } p=0, \\ 0 & \text { if } p>0 .\end{cases}
$$

Theorem 5.2. Let $(W, S)$ be a Coxeter group and $\mathcal{N}$ its nerve. Then

$$
\operatorname{vcd} W=\max _{\text {char } \mathbb{k}}\{\operatorname{reg} \mathbb{k}[\mathcal{N}]\} .
$$

Proof. In Section 2, equations (3) and (4) present interpretations of both invariants in terms of the reduced simplicial cohomology of $\mathcal{N}$, namely

$$
\begin{aligned}
\operatorname{vcd} W & =\max \left\{i: \widetilde{H}^{i-1}\left(\left.\mathcal{N}\right|_{S \backslash \sigma} ; \mathbb{Z}\right) \neq 0 \text { for some } \sigma \in \mathcal{N}\right\}, \\
\operatorname{reg} \mathbb{k}[\mathcal{N}] & =\max \left\{i: \widetilde{H}^{i-1}\left(\left.\mathcal{N}\right|_{U} ; \mathbb{k}\right) \neq 0 \text { for some } U \subseteq S\right\} .
\end{aligned}
$$

Therefore the result is a consequence of the following claim.

Claim. Let $\Delta$ be a simplicial complex on $V=\{0, \ldots, n\}$ and $A$ be a ring. Then

$$
\begin{aligned}
& \max \left\{i: H^{i}\left(\left.\Delta\right|_{V \backslash \sigma} ; A\right) \neq 0 \text { for some } \sigma \in \Delta\right\}= \\
& \quad=\max \left\{i: H^{i}\left(\left.\Delta\right|_{V^{\prime}} ; A\right) \neq 0 \text { for some } V^{\prime} \subseteq V\right\} .
\end{aligned}
$$

Clearly the left-hand side is less than or equal to the the right-hand side. To see that equality holds, let $r$ be the maximum on the right and choose $V^{\prime} \subseteq V$ such that $H^{r}\left(\left.\Delta\right|_{V^{\prime}} ; A\right) \neq 0$. If $V \backslash V^{\prime} \in \Delta$ we have nothing to prove, so assume that $V \backslash V^{\prime} \notin \Delta$ (in particular $\left|V \backslash V^{\prime}\right| \geq 2$ ). We can (and will) also assume that $H^{i}\left(\left.\Delta\right|_{U} ; A\right)=0$ for all $i \geq r$ and $V^{\prime} \subsetneq U \subseteq V$. 
After a potential renumbering we can assume that $V \backslash V^{\prime}=\{0, \ldots, s\}$. For any $i \in\{0, \ldots, s\}$, let $\Delta^{i}=\left.\Delta\right|_{V \backslash\{i\}}$ and consider the double complex defined in (6). By Lemma $5.1\left({ }^{I} E_{r}^{p, q}\right)$ stabilizes at the second page and

$$
H^{p}\left(\cup_{i=0}^{s} \Delta^{i} ; A\right)={ }^{I} E_{2}^{p, 0}={ }^{I} E_{\infty}^{p, 0}={ }^{I} E^{p} .
$$

Since $\{0, \ldots, s\}$ is not a face of $\Delta$, we have $\Delta=\cup_{i=0}^{s} \Delta^{i}$. Now consider the spectral sequence $\left({ }^{I I} E_{r}^{p, q}\right)$. From the maximality assumption on $V^{\prime}$ it follows that

$$
H^{r}\left(\left.\Delta\right|_{V^{\prime}} ; A\right)=H^{r}\left(\Delta^{0, \ldots, s} ; A\right)={ }^{I I} E_{2}^{r, s} .
$$

In particular, if $r^{\prime}>r$ or $s^{\prime}>s$, then ${ }^{I I} E_{2}^{r^{\prime}, s^{\prime}}=0$, since it is a subquotient of

$$
\bigoplus_{0 \leq a_{0}<\ldots<a_{s^{\prime}} \leq s} H^{r^{\prime}}\left(\Delta^{a_{0}, \ldots, a_{s^{\prime}}} ; A\right)=0 .
$$

We have ${ }^{I I} E_{2}^{r, s}={ }^{I I} E_{\infty}^{r, s}={ }^{I I} E^{r+s}$ from which we conclude that

$$
H^{r+s}(\Delta ; A)={ }^{I} E^{r+s}={ }^{I I} E^{r+s}=H^{r}\left(\left.\Delta\right|_{V^{\prime}} ; A\right) \neq 0 .
$$

Since $s>0$ (because $\left|V \backslash V^{\prime}\right| \geq 2$ ), we obtain a contradiction to the maximality of $r$ and $V^{\prime}$.

\section{Arbitrary large Regularity With property $N_{p}$}

We now prove Theorem 6.11. To this end, for each $k$-large simplicial complex $\Delta$ we construct a $k$-large simplicial complex $\mathrm{S}(\Delta, k)$ such that, in characteristic zero, $\operatorname{reg} \mathbb{k}[\mathrm{S}(\Delta, k)]=\operatorname{reg} \mathbb{k}[\Delta]+1$ (Lemma 6.6). This uses a construction based on a detour through geometric group theory and is inspired by the work of Osajda [Osa13b, Section 4].

We need to make a few definitions. The first turns a cell complex into a simplicial complex.

Definition 6.1. The thickening of a convex cell complex $\mathcal{P}$ is the simplicial complex $\operatorname{Th}(\mathcal{P})$, with the same vertex set as $\mathcal{P}$, obtained by turning all cells into simplices. In particular, $\left\{v_{1}, \ldots, v_{s}\right\}$ is a face of $\operatorname{Th}(\mathcal{P})$, if there is a face of $\mathcal{P}$ that contains $\left\{v_{1}, \ldots, v_{s}\right\}$.

Example 6.2. The thickening of the $d$-dimensional cube is the $\left(2^{d}-1\right)$-simplex.

The thickening induces a distance between the vertices of a convex cell complex that counts the minimal number of maximal cells one needs to pass to get from one vertex to another. Namely, for two vertices $v, w \in \mathcal{P}$, the distance $d(v, w)$ is the length of a shortest path connecting $v$ and $w$ in the 1-skeleton of the thickening $\operatorname{Th}(\mathcal{P})$.

A step in our construction is taking a finite quotient of an infinite cubical complex. We clarify here how this is intended. Let $G$ be a group acting on the vertex set $V(\mathcal{P})$ of a convex cell complex $\mathcal{P}$ such that for every face $F=\left\{v_{1}, \ldots, v_{k}\right\} \in \mathcal{P}$ and every $g \in G$ we have

$$
g \cdot F=\left\{g \cdot v_{1}, \ldots, g \cdot v_{k}\right\} \in \mathcal{P} .
$$

This induces an action of $G$ on $\mathcal{P}$. The displacement of the action of $G$ on $\mathcal{P}$ is the minimum distance between the elements in the orbit of a vertex. We can take the quotient $\mathcal{P} / G$, which is in general only a set.

Remark 6.3. If the displacement of the action is at least 2 , then $\mathcal{P} / G$ is a poset with the inclusion given by $\widehat{F^{\prime}} \subseteq \widehat{F}$ if there exists $g \in G$ such that $g \cdot F^{\prime} \subseteq F$. If the displacement of the action is at least 3 , then $\mathcal{P} / G$ is a convex cell complex. 
An example of such a group action is that of the subgroup of some Coxeter group on the vertices of the Davis complex. In this case the displacement of the action coincides with the displacement of the subgroup as defined below.

Definition 6.4. Let $W$ be a Coxeter group. The displacement of an element $w \in W$ is the distance $d(e, w)$ of $w$ to the identity in the (1-skeleton of the) thickening $\operatorname{Th}(\Sigma)$. The displacement of a subgroup $H \subset W$ is the minimal displacement among its nontrivial elements.

Let $\Delta$ be a $k$-large simplicial complex for an integer $k \geq 4$. We introduce an iterative construction which produces a new $k$-large simplicial complex $\mathrm{S}(\Delta, k)$. It works as follows.

(1) Let $W$ be the right-angled Coxeter group with nerve $\Delta$.

(2) Let $\Sigma$ be the Davis complex of $W$.

(3) Let $Y=\operatorname{Th}(\Sigma)$ be the thickening of $\Sigma$.

(4) Pick a torsion-free finite index subgroup $H \subset W$ with displacement at least $k$.

(5) Let $\mathrm{S}(\Delta, k)$ be the quotient $Y / H$.

Since $\Delta$ is flag, there is a right-angled Coxeter group $\mathcal{W}(\Delta)$ as described in Remark 2.11. The group $H$ in 4 exists because $W$ is virtually torsion free [Dav08, Corollary D.1.4] and residually finite [Dav08, Section 14.1]. In Section 7, we take a constructive approach and find a concrete $H$ using representations of $W$ in $\mathrm{GL}_{n}(\mathbb{Z})$. The resulting complex $\mathrm{S}(\Delta, k)$ evidently depends on the choice of $H$ in step 4. However, the desired properties of $\mathrm{S}(\Delta, k)$, such as Lemma 6.6, do not depend on this choice.

Lemma 6.5. In the above situation, $2^{(\Delta)}=\operatorname{lk}_{\mathrm{S}(\Delta, k)} v$ for any vertex $v$.

Proof. After unraveling definitions, it is visible that if $\Sigma$ is a cubical complex and $v \in \Sigma$ is a vertex, then $2^{\left(\mathrm{l}_{\Sigma} v\right)}=\mathrm{lk}_{\mathrm{Th}(\Sigma)} v$. If $\Sigma$ is the Davis complex of a right-angled Coxeter group with nerve $\Delta$, then by Remark $2.15, \mathrm{lk}_{\Sigma} v=\Delta$ for any vertex $v \in \Sigma$.

Lemma 6.6. If $\mathbb{k}$ is a field of characteristic zero and $k \geq 4$, then $\operatorname{reg} \mathbb{k}[\mathrm{S}(\Delta, k)]=\operatorname{reg} \mathbb{k}[\Delta]+1$.

Proof. By the previous lemma $2^{(\Delta)}=\operatorname{lk}_{\mathrm{S}(\Delta, k)} v$ for any vertex $v$, and by Proposition 3.2 there exists a vertex $v$ such that $\operatorname{reg} \mathbb{k}\left[\operatorname{lk}_{\mathrm{S}(\Delta, k)} v\right] \geq \operatorname{reg} \mathbb{k}[\mathrm{S}(\Delta, k)]-1$. Since reg $\mathbb{k}\left[2^{(\Delta)}\right]=\operatorname{reg} \mathbb{k}[\Delta]$ by Proposition 3.5, it follows that $\operatorname{reg} \mathbb{k}[\mathrm{S}(\Delta, k)] \leq \operatorname{reg} \mathbb{k}[\Delta]+1$.

To show reg $\mathbb{k}[\mathrm{S}(\Delta, k)] \geq \operatorname{reg} \mathbb{k}[\Delta]+1$, let $X=\Sigma / H$. Then $\mathrm{S}(\Delta, k)$ is the thickening of $X$. By Hochster's formula for graded Betti numbers and (8), we have that

$$
\operatorname{reg} \mathbb{k}[\Delta]=\max \left\{i: \widetilde{H}^{i-1}(\Delta \backslash \sigma ; \mathbb{k}) \neq 0 \text { for some } \sigma \in \Delta\right\} .
$$

Let $r=\operatorname{reg} \mathbb{k}[\Delta]$, and fix $\sigma \in \Delta$ for which $\widetilde{H}^{r-1}(\Delta \backslash \sigma ; \mathbb{k}) \neq 0$. From now on the argument goes on the same lines of the proof leading to [Osa13a, Lemma 4.5]. With the same notation used there, $\Delta \backslash \sigma$ deformation retracts onto $K^{S \backslash \sigma}$, where $K$ is the subcomplex of $\Sigma$ induced by the spherical words (including the identity) and, for any subset of generators $T \subseteq S, K^{T}$ is the subcomplex induced by the spherical words containing some element of $T$. So we have

$$
\widetilde{H}^{r}\left(K, K^{S \backslash \sigma} ; \mathbb{k}\right) \neq 0 .
$$

Osajda produces a map of $\mathbb{k}$-vector spaces from the cocycles $Z^{r}\left(K, K^{S} ; \mathbb{k}\right)$ to the cocycles $Z^{r}(X ; \mathbb{k})$. This uses the assumption $\operatorname{char}(\mathbb{k})=0$. One can check that the same rule defines a map of $\mathbb{k}$-vector spaces $Z^{r}\left(K, K^{S \backslash \sigma} ; \mathbb{k}\right) \rightarrow Z^{r}(X \backslash A ; \mathbb{k})$, where $A=\{\widehat{w \sigma}: w \in W\}$ and $\widehat{w \sigma}$ is 
the class in $X$ of $w \sigma \in \Sigma$. By the same argument used in [Osa13a, Lemma 4.5], the above map induces an injection

$$
\widetilde{H}^{r}\left(K, K^{S \backslash \sigma} ; \mathbb{k}\right) \hookrightarrow \widetilde{H}^{r}(X \backslash A ; \mathbb{k}),
$$

in particular $\widetilde{H}^{r}(X \backslash A ; \mathbb{k})$ is not zero. By [Mun84, Lemma 70.1], $\widetilde{H}^{k}(X \backslash A ; \mathbb{k}) \cong \widetilde{H}^{r}\left(X_{B} ; \mathbb{k}\right)$, where $B$ are the vertices of $X$ which are not in $\widehat{w \sigma}$ for any $w \in W$. Finally, the thickening of $X_{B}$ is exactly $\mathrm{S}(\Delta, k)_{B}$, so

$$
\widetilde{H}^{r}\left(\mathrm{~S}(\Delta, k)_{B} ; \mathbb{k}\right) \neq 0 .
$$

By Hochster's formula for graded Betti numbers reg $\mathbb{k}[\mathrm{S}(\Delta, k)] \geq r+1$.

Remark 6.7. In the definition of cohomological dimension, $\mathbb{Z}$ could be replaced by a field $\mathbb{k}$ of characteristic zero. The resulting notion of virtual rational cohomological dimension $\operatorname{vcd}_{\mathbb{Q}} W$ of a virtually torsion free group $W$ does not depend on the choice of the field. This notion however, differs from virtual cohomological dimension. Lemma 6.6, together with Hochster's formula for graded Betti numbers and (8), implies that

$$
\operatorname{vcd}_{\mathbb{Q}} \mathcal{W}(\mathrm{S}(\Delta, k))=\operatorname{vcd}_{\mathbb{Q}} \mathcal{W}(\Delta)+1
$$

This conclusion for ved does not follow from Lemma 6.6 because of the assumptions on $\mathbb{k}$.

Lemma 6.8. If a cubical complex is locally $k$-large, then its thickening is locally $k$-large.

Proof. Let $\Sigma$ be a locally $k$-large cubical complex. As in the proof of Lemma 6.5, each vertex link $\mathrm{lk}_{\mathrm{Th}(\Sigma)} v$ is equal to $2^{\left(\mathrm{lk}_{\Sigma} v\right)}$. By a result of Haglund, a simplicial complex is $k$-large if and only if its face complex is $k$-large [JŚ10, Proposition B.1].

A proof of the Lemma 6.8 also appears in [Osa13a, Lemma 6.7].

Lemma 6.9. Let $\Sigma$ be the Davis complex of $\mathcal{W}(\Delta)$, where $\Delta$ is $k$-large for $k \geq 4$. Then $\operatorname{Th}(\Sigma)$ is k-large.

Proof. The Davis complex $\Sigma$ is a deformation retract of its thickening $\operatorname{Th}(\Sigma)$ and in particular has the same homotopy type. Therefore $\operatorname{Th}(\Sigma)$ is simply connected. By Lemma $6.8 \operatorname{Th}(\Sigma)$ is locally $k$-large. According to [JŚ06, Corollary 1.5], a simplicial complex is $k$-large if and only if all links are $k$-large and the systole (the length of the shortest non-contractible loop in the complex) is at least $k$. Since there are no non-contractible loops, the proof is complete.

When forming the quotient of the thickening of the Davis complex modulo the finite-index torsion-free subgroup $H \subset W$ in step 5 of the construction, cycles are created. The quotient by a group of displacement $k$ creates cycles of length $k$. By Remark $6.3, k \geq 4$ implies the quotient is simplicial complex.

Lemma 6.10. Let $\Sigma$ be the Davis complex of $\mathcal{W}(\Delta)$, where $\Delta$ is $k$-large for $k \geq 4$. If $H \subset \mathcal{W}(\Delta)$ is a torsion-free subgroup of displacement at least $k$, then $\operatorname{Th}(\Sigma) / H$ is $k$-large.

Proof. If $C \in \operatorname{Th}(\Sigma) / H$ is a cycle of length $l<k$, then it consists of disjoint orbits and thus there is a cycle of length $l$ in $\operatorname{Th}(\Sigma)$. This is impossible since by Lemma $6.9 \operatorname{Th}(\Sigma)$ is $k$-large.

We are now ready to prove the two main results of this section. 
Theorem 6.11. Let $I=I_{\Delta} \subseteq R=\mathbb{k}\left[x_{1}, \ldots, x_{n}\right]$ be a square-free quadratic monomial ideal. If the characteristic of $\mathbb{k}$ is zero, then there exists a positive integer $N$ and a square-free monomial ideal $I^{\prime}=I_{\Delta^{\prime}} \subseteq R^{\prime}=\mathbb{k}\left[y_{1}, \ldots, y_{N}\right]$ such that:

i) $\operatorname{reg} R^{\prime} / I^{\prime}=\operatorname{reg} R / I+1$;

ii) index $R^{\prime} / I^{\prime}=$ index $R / I$;

iii) For each vertex $v$ of $\Delta^{\prime}, \mathrm{lk}_{\Delta^{\prime}} v=2^{(\Delta)}$.

Proof. Let $p=\operatorname{index} R / I$. Since $I=I_{\Delta}$ is quadratic, $p \geq 1$. Let $\Delta^{\prime}=\mathrm{S}(\Delta, p+3)$. The first item is Lemma 6.6. The third item is Lemma 6.5. For the second item, Lemma 6.9 implies that index $R^{\prime} / I^{\prime} \geq p$. If index $R^{\prime} / I^{\prime}>p$, then index $\mathbb{k}\left[2^{(\Delta)}\right]>p$ since if there are no induced $(p+3)$ cycles, then no link in $\Delta$ has an induced $(p+3)$-cycle. Furthermore, by [JŚ10, Proposition B.1], index $\mathbb{k}\left[2^{(\Delta)}\right]=\operatorname{index} \mathbb{k}[\Delta]=\operatorname{index} R / I$.

Corollary 6.12. For any positive integers $p$ and $r$, there exists a square-free monomial ideal $I \subseteq R=\mathbb{k}\left[x_{1}, \ldots, x_{N(p, r)}\right]$, such that $R / I$ satisfies $N_{p}$ and $\operatorname{reg} R / I=r$.

Proof. Let $\Delta_{2}$ be the $(p+3)$-cycle, and inductively $\Delta_{r}=\mathrm{S}\left(\Delta_{r-1}, p+3\right)$. Then $\Delta_{r}$ satisfies the conditions of the corollary if char $\mathbb{k}=0$. To see that the construction is independent of the field, assume that for some $\mathbb{k}, \operatorname{reg} \mathbb{k}\left[\Delta_{r}\right]>\operatorname{reg} \mathbb{Q}\left[\Delta_{r}\right]$. By Lemma 6.5, Proposition 3.2, and Proposition 3.5, $\operatorname{reg} \mathbb{k}\left[\Delta_{r-1}\right]>\operatorname{reg} \mathbb{Q}\left[\Delta_{r-1}\right]$ and inductively reg $\mathbb{k}\left[\Delta_{2}\right]>\operatorname{reg} \mathbb{Q}\left[\Delta_{2}\right]$ which is not the case.

Remark 6.13. In [JŚ06, Corollary 19.2], Januszkiewicz and Świątkowski proved, for any $k \geq 6$ and $d \in \mathbb{N}$, the existence of a $k$-large orientable $d$-dimensional pseudomanifold. Together with [JŚ03, Theorem 1] this could be used to give a shorter proof of Corollary 6.12. We feel that such a proof would have been less insightful for commutative algebra.

Remark 6.14. The results in this section can also be used to strengthen a result of Nevo and Peeva who studied a question of Francisco, Hà and Van Tuyl. The latter noticed (unpublished) that if $I \subset R$ is a quadratic square-free monomial ideal such that $I^{s}$ has a linear resolution for all $s \geq 2$, then $R / I$ satisfies $N_{2}$, and wondered if the converse was true. In [NP13, Counterexample 1.10] Nevo and Peeva gave a square-free monomial ideal $I \subset R$ such that $R / I$ has property $N_{2}$ but $I^{2}$ does not have a linear resolution. Using our results, this can be extended to $N_{p}$ and any power as follows.

Corollary 6.15. For any integers $p, t \geq 2$ there exists a square-free monomial ideal $I \subset R$ such that $R / I$ has property $N_{p}$ and $I^{s}$ does not have a linear resolution for all $1 \leq s \leq t$.

Proof. Set $r=2 t$ and choose $I \subset R$ as in Corollary 6.12. Then $\operatorname{reg}(R / I)=2 t$, and $\operatorname{reg}\left(R / I^{s}\right) \geq$ $2 t$ for all $s \geq 1$ by Proposition 3.3.

Question 1.11 in [NP13] asks whether $I_{\Delta}^{s}$ has a linear resolution for $s \gg 0$ whenever $\mathbb{k}[\Delta]$ satisfies $N_{2}$. It remains open and the construction yielding Theorem 6.11 provides examples worth testing. For an experimental investigation with computer algebra, the number of variables involved would need a vast improvement, though. 


\section{Counting the number of vertices of $\mathrm{S}(\Delta, k)$}

For complexity theory in commutative algebra a bound on the number of variables $N(r, p)$ in Corollary 6.12 is necessary. We now derive such a bound by controlling the choice of the torsion-free subgroup $H$ in step 4 of the construction of $\mathrm{S}(\Delta, k)$.

Each Coxeter group $W$ can be embedded in $\mathrm{GL}_{n}(\mathbb{R})$ by means of its canonical representation $\rho: W \rightarrow \mathrm{GL}_{n}(\mathbb{R})$ [Dav08, Corollary 6.12.4]. This representation starts from the cosine matrix $C=\left(c_{i j}\right)_{i j}$ of a Coxeter system whose entries are $c_{i j}=-\cos \left(\pi / m_{i j}\right)$. A generator $s_{i}$ is represented by the linear map $\rho\left(s_{i}\right): x \mapsto x-2 \sum_{j} c_{i j} x_{j} e_{i}$. As the order of every product of generators is 2 or $\infty$, right-angled Coxeter groups embed also in $\mathrm{GL}_{n}(\mathbb{Z})$. More specifically, since the cosine matrix has entries only $-1,0,1$, the canonical representation matrices use only $0, \pm 1,2$. An easy computation using the definition of the linear map for one generator and $\cos (\pi / 2)=0$ shows that whenever $w=s_{i_{1}} \cdots s_{i_{l}}$ is a spherical word, then it is represented by the linear map

$$
\rho(w): x \mapsto x-2 \sum_{j} c_{i_{1} j} x_{j} e_{i_{1}}-\cdots-2 \sum_{j} c_{i_{l} j} x_{j} e_{i_{l}}
$$

We thus showed a simple fact about the entries of $\rho(w)$.

Lemma 7.1. Let $\mathcal{W}(\Delta)$ be a right-angled Coxeter group with nerve $\Delta$ and $d=\operatorname{dim} \Delta$. For each spherical word $w \in \mathcal{W}(\Delta)$ of length $l$, the matrix $\rho(w)$ uses only $0, \pm 1,2$ for its entries and each of its columns has at most l entries equal to two.

We employ the projection $\mathrm{GL}_{n}(\mathbb{Z}) \rightarrow \mathrm{GL}_{n}(\mathbb{Z} / m \mathbb{Z})$ to find finite-index torsion-free subgroups $H$ as in step 4 of the construction in Section 6 , so that the size of $\mathrm{S}(\Delta, k)$ can be controlled. To preserve $k$-largeness, we need to choose $m$ so that no words of displacement $<k$ reduce to the identity modulo $m$. This requires information about the orders of elements of $\mathrm{GL}_{n}(\mathbb{Z} / m \mathbb{Z})$.

Fix $k>4$ and a $k$-large simplicial complex $\Delta$ of dimension $d$ with $n$ vertices. For any $m \geq 2$ consider the canonical homomorphism

$$
\pi_{m}: \mathrm{GL}_{n}(\mathbb{Z}) \rightarrow \mathrm{GL}_{n}(\mathbb{Z} / m \mathbb{Z}) .
$$

Denote $\Gamma_{m}=\operatorname{Ker}\left(\pi_{m}\right)$ and let $\Xi_{m}=\Gamma_{m} \cap \rho(\mathcal{W}(\Delta)) \subseteq \rho(\mathcal{W}(\Delta))$ be the subgroup of $\rho(\mathcal{W}(\Delta))$ that lies in the kernel of $\pi_{m}$.

Lemma 7.2. $\Xi_{m}$ is torsion free if $m>2$.

Proof. It is well known that any torsion element in a right-angled Coxeter group has order two and is in fact conjugate to a spherical word. Let $w \in \Xi_{m}$ be an involution and write $w=g^{-1} s g$ with some spherical word $s$ and $g \in \mathcal{W}(\Delta)$. Then $1=\pi_{m}(w)=\pi_{m}(g)^{-1} \pi_{m}(s) \pi_{m}(g)$ implies $\pi_{m}(s)=1$ which for $m>2$ implies $s=1$ (by Lemma 7.1) and finally $w=1$.

The subgroup to be used in step 4 is $H(m)=\rho^{-1}\left(\Xi_{m}\right)$. Let $w \in \mathcal{W}(\Delta)$. As a function of the displacement and the dimension $d$ of $\Delta$, we determine an upper bound on $a(w)=$ $\max \left\{\left|\rho(w)_{i, j}\right|: 1 \leq i, j \leq n\right\}$, the maximum absolute value of the entries of the corresponding $\operatorname{matrix} \rho(w) \in \mathrm{GL}_{n}(\mathbb{Z})$.

Lemma 7.3. Let $w$ be a word of displacement less than $k$, then $a(w)<(2 d+3)^{k-1}$. 
Proof. A word of displacement less than $k$ is a product of at most $k-1$ spherical words. When $w$ is a spherical word, it has length at most $d+1$, and thus each column of $\rho(w)$ has at most $d+1$ entries 2 and one entry 1 by Lemma 7.1. This yields the recursion $a(w s) \leq(2 d+3) a(w)$. Since $a(s)=2$ for any spherical word, the bound follows.

Our aim is to pick an integer $m$ so that any word in $H(m)$ has displacement at least $p+3$. Lemma 7.3 shows that $m=(2 d+3)^{p+2}$ is sufficient. Given $m$, the number of vertices of $\mathrm{S}(\Delta, p)$ is bounded by the size of $\mathrm{GL}_{n}(\mathbb{Z} / m \mathbb{Z})$ which is of the order $m^{n^{2}}$. Iterating the construction of $\mathrm{S}(\Delta, p)$, we achieve the desired bound for the number of variables needed in Corollary 6.12. To write it, we use Knuth's up arrow notation [Knu76] which is convenient for iterative constructions. Fortunately we can limit ourselves to two up arrows which represent power towers. Specifically, $a \uparrow \uparrow b$ means $a^{a^{a^{\cdots}}}$ exactly $b$ times.

Theorem 7.4. For all $p$, there exists a family of ideals indexed by $r$ realizing Corollary 6.12 with

$$
N(p, r+1)<(2(2 \uparrow \uparrow(r-1))+1)^{(p+2) N(p, r)^{2}} .
$$

Furthermore, if $c_{p}$ is the smallest integer such that $2 \uparrow \uparrow c_{p}>p+2$, then

$$
N(p, r+1)<2 \uparrow \uparrow\left(r\left(r+c_{p}\right)\right) .
$$

Proof. Let $\Delta_{2}$ be the $(p+3)$-cycle which implies $N(p, 2)=p+3$. Let $\Delta_{r+1}=\mathrm{S}\left(\Delta_{r}, p\right)$, where the subgroup in step 4 is chosen as $H\left(m_{r+1}\right)$ with $m_{r+1}=\left(2 d_{r}+1\right)^{p+2}$. Here $d_{r}=\operatorname{dim} \Delta_{r}+1$ and thus $d_{2}=2$. We have the recursion $d_{r+1}=2^{d_{r}}$, which yields $d_{r}=2 \uparrow \uparrow(r-1)$. The number of vertices of $\Delta_{r+1}$ is bounded by the order of $\mathrm{GL}_{n}\left(\mathbb{Z} / m_{r+1} \mathbb{Z}\right)$. Estimating this order as $m_{r+1}^{N(r, p)^{2}}$ we obtain the recursive bound.

For the second part we use the fact that removing parenthesis from a power tower does not make the expression smaller by generalizations of $\left(2^{2}\right)^{\left(2^{2}\right)}<2^{2^{2^{2}}}$. We thus get

$$
\begin{aligned}
N(p, r+1) & <(2 \uparrow \uparrow r)^{(p+2) N(p, r)^{2}} \\
& <(2 \uparrow \uparrow r)^{\left(2 \uparrow \uparrow c_{p}\right) N(p, r)^{2}} \\
& <\left(2 \uparrow \uparrow\left(r+c_{p}\right)\right)^{N(p, r)^{2}} .
\end{aligned}
$$

Now by a simple induction, the structure of the expression on the right is continued exponentiation of 2 for at most $r\left(r+c_{p}\right)$ times, but with certain parenthesis inside the tower. Removing the parentheses we conclude.

We hope that the bound in Theorem 7.4 can be improved significantly. To justify this hope we illustrate vast improvements in a simple example. Let $\Delta$ be the 5 -cycle. The right-angled Coxeter group with nerve $\Delta$ has the following Coxeter and cosine matrices

$$
\left(\begin{array}{ccccc}
1 & 2 & \infty & \infty & 2 \\
2 & 1 & 2 & \infty & \infty \\
\infty & 2 & 1 & 2 & \infty \\
\infty & \infty & 2 & 1 & 2 \\
2 & \infty & \infty & 2 & 1
\end{array}\right), \quad C=\left(\begin{array}{rrrrr}
1 & 0 & -1 & -1 & 0 \\
0 & 1 & 0 & -1 & -1 \\
-1 & 0 & 1 & 0 & -1 \\
-1 & -1 & 0 & 1 & 0 \\
0 & -1 & -1 & 0 & 1
\end{array}\right) .
$$


The generators of the standard representation of this Coxeter group are

$$
s_{1} \mapsto\left(\begin{array}{rrrrr}
-1 & 0 & 2 & 2 & 0 \\
0 & 1 & 0 & 0 & 0 \\
0 & 0 & 1 & 0 & 0 \\
0 & 0 & 0 & 1 & 0 \\
0 & 0 & 0 & 0 & 1
\end{array}\right) \quad \ldots \quad s_{5} \mapsto\left(\begin{array}{rrrrr}
1 & 0 & 0 & 0 & 0 \\
0 & 1 & 0 & 0 & 0 \\
0 & 0 & 1 & 0 & 0 \\
0 & 0 & 0 & 1 & 0 \\
0 & 2 & 2 & 0 & -1
\end{array}\right) .
$$

Table 1 gives the maximum absolute value of entries of words of length $l$ in $\mathcal{W}(\Delta)$. It shows that the prime number $p=1811$ would certainly suffice to guarantee that no word of displacement $\leq 5$ (which all have length $\leq 10$ ) is in the kernel of the reduction modulo $p$. However, it can be

\begin{tabular}{|l||c|c|c|c|c|c|c|c|c|c|}
\hline word length & 1 & 2 & 3 & 4 & 5 & 6 & 7 & 8 & 9 & 10 \\
\hline entry size & 2 & 4 & 8 & 18 & 39 & 84 & 180 & 388 & 836 & 1801 \\
\hline
\end{tabular}

TABLE 1. Entry sizes in words

checked algorithmically (we used the Coxeter group functionality in SAGE $\left[\mathrm{S}^{+} 16\right]$ ) that no word of length at most 10 is in the kernel of the reduction modulo 7 . In the reduction modulo 5, however, $\left(s_{1} s_{3}\right)^{5}$ maps to the identity. We also checked words of length 12 for the Coxeter group corresponding to the heptagon. There 7 is not large enough, as for example $\left(s_{1} s_{3} s_{1} s_{5}\right)^{3}$ goes to the identity.

In the example of the 5 -cycle, the bound derived in Theorem 7.4 yields $N(2,3)<5^{100}$, while using $m=7$ yields $N(2,3)<7^{25}$. In contrast one can exhibit a 5-large triangulation of a 2-sphere with 12 vertices. Nevertheless, a good understanding of representations of Coxeter groups in finite characteristic should yield better estimates than Theorem 7.4.

The integer $m_{r}$ used in the recursive construction of $\Delta_{r}$ in Theorem 7.4 currently depends on the dimension which grows very quickly. It is conceivable that for each $p$ there is a uniform bound, independent of $r$.

Question 7.5. Is there a bound for the integer $m_{r}$ that depends only on $p$ and not on $r$ ?

\section{REFERENCES}

[ACI13] Luchezar L Avramov, Aldo Conca, and Srikanth B Iyengar, Subadditivity of syzygies of Koszul algebras, Mathematische Annalen 361 (2013), no. 1-2, 511-534.

[AH16] T. Ananyan and M. Hochster, Small subalgebras of polynomial rings and Stillman's conjecture, preprint, arXiv:1610.09268 (2016).

[BS88] David Bayer and Michael Stillman, On the complexity of computing syzygies, Journal of Symbolic Computation 6 (1988), no. 2, 135-147.

[BS13] M. P. Brodmann and R. Y. Sharp, Local cohomology, second ed., Cambridge Studies in Advanced Mathematics, vol. 136, Cambridge University Press, Cambridge, 2013, An algebraic introduction with geometric applications.

$\left[\mathrm{CCM}^{+} 19\right]$ Giulio Caviglia, Marc Chardin, Jason McCullough, Irena Peeva, and Matteo Varbaro, Regularity of prime ideals, Mathematische Zeitschrift 291 (2019), 421-435.

[CKV16] Alexandru Constantinescu, Thomas Kahle, and Matteo Varbaro, Linear syzygies, flag complexes, and regularity, Collectanea Mathematica 67 (2016), no. 3, 357-362.

[Cox35] H. S. M. Coxeter, The complete enumeration of finite groups of the form $R_{i}^{2}=\left(R_{i} R_{j}\right)^{k_{i j}}=1$, Journal of the London Mathematical Society s1-10 (1935), no. 1, 21-25. 
[CS05] Giulio Caviglia and Enrico Sbarra, Characteristic-free bounds for the Castelnuovo-Mumford regularity, Compositio Mathematica 141 (2005), no. 6, 1365-1373.

[Dav08] Michael Davis, The geometry and topology of Coxeter groups, vol. 32, Princeton University Press, 2008.

[DHS13] Hailong Dao, Craig Huneke, and Jay Schweig, Bounds on the regularity and projective dimension of ideals associated to graphs, Journal of Algebraic Combinatorics 38 (2013), no. 1, 37-55.

[DJ91] Michael Davis and Tadeusz Januszkiewicz, Convex polytopes, Coxeter orbifolds and torus actions, Duke Math. J 62 (1991), no. 2, 417-451.

[EG84] David Eisenbud and Shiro Goto, Linear free resolutions and minimal multiplicity, Journal of Algebra 88 (1984), no. 1, 89-133.

[EGHP05] David Eisenbud, Mark Green, Klaus Hulek, and Sorin Popescu, Restricting linear syzygies: algebra and geometry, Compositio Mathematica 141 (2005), no. 6, 1460-1478.

[Eis95] David Eisenbud, Commutative algebra with a view toward algebraic geometry, GTM, vol. 150, Springer Verlag, New York, 1995.

[ES09] David Eisenbud and Frank-Olaf Schreyer, Betti numbers of graded modules and cohomology of vector bundles, Journal of the American Mathematical Society 22 (2009), no. 3, 859-888.

[GL86] Mark Green and Robert Lazarsfeld, On the projective normality of complete linear series on an algebraic curve, Inventiones mathematicae 83 (1986), no. 1, 73-90.

[GM03] S. I. Gelfand and Yu. I. Manin, Methods of homological algebra, second ed., Springer Monographs in Mathematics, Springer, 2003.

[JŚ03] Tadeusz Januszkiewicz and Jacek Świątkowski, Hyperbolic Coxeter groups of large dimension, Commentarii Mathematici Helvetici 78 (2003), no. 3, 555-583.

[JŚ06] _ Simplicial nonpositive curvature, Pub. Math. IHES 104 (2006), no. 1, 1-85.

[JŚ10] _ N N Non-positively curved developments of billiards, Journal of Topology 3 (2010), no. 1, 63-80.

[Knu76] Donald Ervin Knuth, Mathematics and computer science: coping with finiteness, Science 194 (1976), no. 4271, 1235-1242.

[Lyu84] Gennady Lyubeznik, On the local cohomology modules $H_{\mathfrak{A}}^{i}(R)$ for ideals $\mathfrak{A}$ generated by monomials in an R-sequence, Complete intersections (Acireale, 1983), Lecture Notes in Math., vol. 1092, Springer, Berlin, 1984, pp. 214-220.

[MM82] Ernst W. Mayr and Albert A. Meyer, The complexity of the word problems for commutative semigroups and polynomial ideals, Advances in Mathematics 46 (1982), no. 3, 305-329.

[MP18] Jason McCullough and Irena Peeva, Counterexamples to the Eisenbud-Goto regularity conjecture, Journal of the American Mathematical Society 31 (2018), no. 2, 473-496.

[MS05] Ezra Miller and Bernd Sturmfels, Combinatorial commutative algebra, GTM, vol. 227, Springer, Berlin, 2005.

[MS13] Jason McCullough and Alexandra Seceleanu, Bounding projective dimension, Commutative algebra, Springer, 2013, pp. 551-576.

[MSS17] Linquan Ma, Karl Schwede, and Kazuma Shimomoto, Local cohomology of Du Bois singularities and applications to families, Compositio Mathematica 153 (2017), no. 10, 2147-2170.

[Mun84] James R Munkres, Elements of algebraic topology, vol. 2, Addison-Wesley, 1984.

[NP13] Eran Nevo and Irena Peeva, $C_{4}$-free edge ideals, Journal of Algebraic Combinatorics 37 (2013), no. 2, $243-248$.

[Osa13a] Damian Osajda, A combinatorial non-positive curvature I: weak systolicity, preprint, arXiv:1305.4661 (2013).

[Osa13b] _ A construction of hyperbolic Coxeter groups, Comment. Math. Helv. 88 (2013), no. 2, 353367.

[PS09] Irena Peeva and Mike Stillman, Open problems on syzygies and Hilbert functions, Journal of Commutative Algebra 1 (2009), no. 1, 159-195.

[S $\left.\mathrm{S}^{+} 16\right] \quad$ W. A. Stein et al., Sage Mathematics Software (Version 7.3), The Sage Development Team, 2016, http://www. sagemath.org.

[Sch09] Karl Schwede, F-injective singularities are Du Bois, Amer. J. Math. 131 (2009), no. 2, 445-473. 
[Ul114] Brooke Ullery, Designer ideals with high Castelnuovo-Mumford regularity, Math. Res. Lett. 21 (2014), $1215-1225$.

Mathematisches Institut, Freie Universität Berlin, Arnimallee 3, 14195 Berlin, Germany $U R L:$ http://userpage.fu-berlin.de/aconstant/Main.html

Fakultät für Mathematik, OvGU Magdeburg, Universitätsplatz 2, 39106 Magdeburg, Germany $U R L:$ http: //www.thomas-kahle.de

Dipartimento di Matematica, Università di Genova, Via Dodecaneso 35, Genova 16146, Italy $U R L:$ http://www.dima.unige.it/ varbaro/ 